\title{
Proficient Empirical Optimization Techniques and Routing Protocols for Internet of Things Networks
}

\author{
Salem Ba Hmaid \\ Research Scholar, Department of CS \\ Rathinam college of Arts \& Science, Coimbatore, India
}

\author{
V. Vasanthi, PhD \\ Department of Information Technology \\ Sri Krishna Adithya College of Arts and Science \\ Bharathiar University, Coimbatore, India
}

\begin{abstract}
The internet of things (IOT) has become a tremendous technology in today world where various objects are connected to each other in order to achieve certain task. According to the statics there will be more than 50 billion devices connected to each other in 2020 and this brings that the internet of things become more useful in the upcoming days . Another concern is that the energy efficient routing protocol is a major concept for making the objects to be connected and exchange their data in the environment .the most important challenges for internet of things are the energy lost while data is transferring from source to distinction. This paper presents most crucial optimization techniques and energy efficient routing protocols that can be used for the internet of things. Paper present the proficient study of the techniques and the energy efficient routing protocols for the internet of things.
\end{abstract}

\section{Keywords}

Internet of things, optimization techniques, energy efficient routing protocol, wireless sensor network

\section{INTRODUCTION}

The internet of things have become one of the most crucial technology which can make the physical devices and virtual machine to be interacted to each other this technology is growing very fast which made many objects being connected and transfer their data to each other via the internet .There are many examples of internet of things, such as smart city, smart, home appliance, smart home, health care, transport, military, agriculture, educational etc. The internet of things is a fast technology growing which uses things and objects to make communication for different processing applications [1]. The wireless sensor networks depends on the multi sensors nodes and number of autonomous that enable the internet of things networks efficiency [2]. The main important challenges in wireless sensor networks which are link failure and energy constraint problems and taking into considerations the packet loss ,less throughput and delay. The main issues are the probably be the routing protocol and the efficient development of multi-constraint protocol [3].other protocols are rely on shortest path and this protocols can be single path routing [4].also the optimal paths are depend on the a single objective and many shortest path not be efficient processing of routing efficient. multi-constraint routing protocol is the main concept that has been created in order to select the path for transmission of the data efficiently[5].the routing protocols are the most important concept in order to send the data packet from source to destinations .there are multiple path and single path routing, the main issue is that the single path routing protocols can be loss the packet because of the traffic congestion or the failures of the link at the time of the expirations of the nodes.so when there is failure in the cluster head selection ,the cluster head cannot be send the data and the result will be data lost.so in order to make sure to send the data ,the fault tolerance concept is used for single path routing protocols[6].

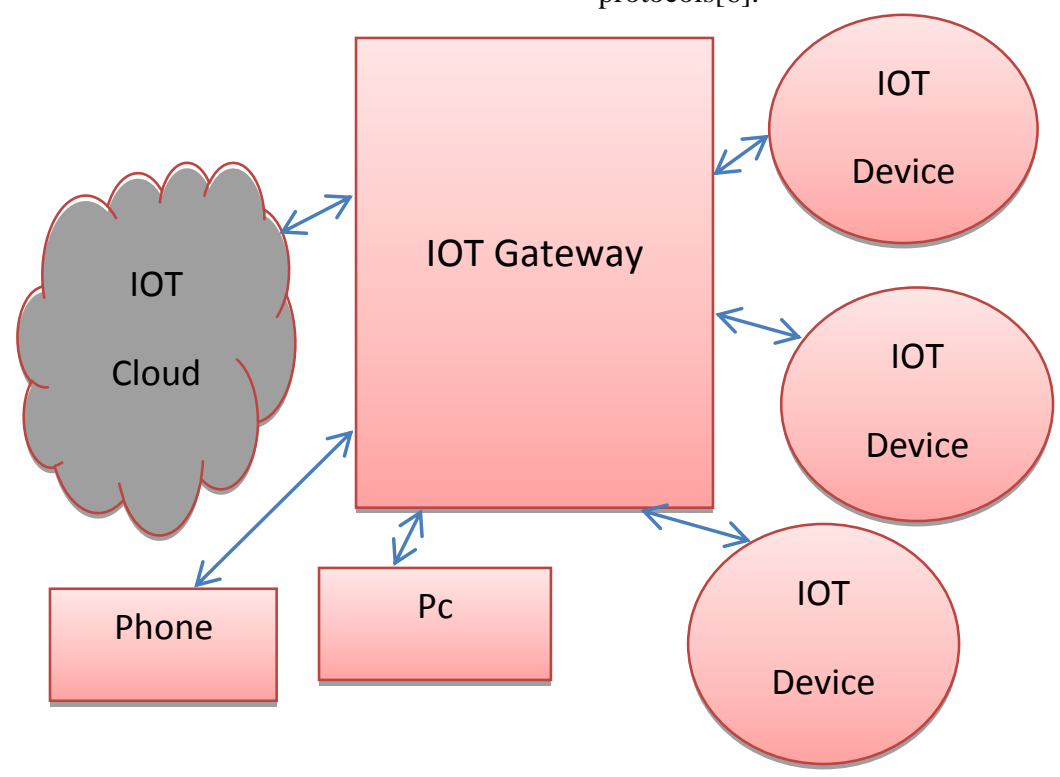

Fig.1. Internet of Things (IoT) 


\section{INTERNET OF THINGS PROTOCOLS}

There are many routing protocols that can be used in the field of internet of things . in this chapter is explained about the recent different routing protocols that have been proposed for the internet of things.

Pan et al. [7] stated the multicast routing issue which contained power computation are more that can decided in which the multicast packets can send.to overcome this issue ,the author have achieved a lightweight geographic multicast routing protocol which can be used for reducing the latency. $\mathrm{He}$ also got detected the drawbacks which is in the existing system such as the nodes of the networks. The main enhancement in this paper is that the path length and link transmission are reduced. The mobility of the nodes are not mentioned and not discussed.

The energy efficiency become very important for internet of things which led to design a routing protocol in order to enhance the energy efficiency .Rani et al. [8] achieved ME$\mathrm{CBCCP}$ optimization algorithm which can get the solution for optimization issues and the scalability in the internet of things .This designed algorithm delivers energy efficiency and the internet of things flexibility. The algorithm compared with existing wireless sensor network and got better performance in time ,scalability and network lifetime.

Tseng et al. [9]designed a mechanism for load balancing which can be used to handle the traffic of bursty in the gateway of the internet of things there is much traffic due to IOT sensor network and that led to more number of nodes increased. To overcome the issue of the Sigsbee's, the multiload balancing routing (MBR)protocol is used. While comparing Zigbee's along with MBR, the multi-load bring less packets of data loss, provides good communication in grid topology. This multiload protocol is good solution for the internet of things.

Qiu et al. [10] presented a sensor networks for internet of things which is called Efficient Tree-based self-organization protocol (ETSP).the protocol which has been proposed is can stay longer in term of the network lifetime and that lead get more energy efficiency.this protocols uses many process such as ,communication ,hop count, child nodes, residual energy and distance.

Shin et al. [11] designed an energy efficient routing to make the utilization of the energy and life of the network better .the new technique is also proposed for the arrangement of local nodes. This routing protocol uses the centroid position in order to select cluster head selection. The selection for the cluster head is done based on the centroid and that made the reduced of the distance long communication. The residual energy is used in the routing protocol for calculation the centroid position. The presented protocol can be fit in order to bring maximum life time of the network . the dead nodes and cluster nodes are the main concern in this routing protocol which has been framed based on the optimization techniques. The drawbacks of proposed routing protocol is not taking the study of the multi-hop path in the consideration and this needs to be extended for further work especially for the located of the base station.

Baker et al. [12] designed an algorithm for multi cloud internet of things for the user request to be satisfying in the energy efficiency. The base cloud, smart are used for calculation this proposed work is reached a good performance based on services number the optimal result is done and energy efficiency are better in this algorithm. Many routing protocols are proposed to reach the reliability.

Qiu et al. [13] presented algorithm for internet of things that can be used in response of emergency applications in IOT. This protocol is improved for the performance of data transmission in term of emergency response and reliability In IOT. this proposed algorithm is used to solve the valid path. This method helps in data transmission and reliability.

Xie et al. [14] proposed the routing approach which is depended on the programming of dynamic. This method is achieved to solve two problem which are the conquer and divide.the algrothim protocol has achieved the performance by decreasing latency, consumption of the resource and fault tolerance. This work should be extend in Dcell Ficonn in order to improve in ARM.

Hasan et al. [15] presented approach for enhancing the communication of the objects and assured the fault tolerance The routing algorithm for bio-inspired particle swarm optimization is achieved to choose the k-disjoint multipath and construct the nodes a

Shu et al. [16] achieved a method for location routing mechanism to make the best communication efficient. The WSN has existing location-based mechanisms and the issue exists with private performance in location mechanisms. The information distribution in the internet of things node neighbours may take the privacy problem communication into consideration.the Hilbert curve encrypted is used for geographic routes for computed.this issue is made the privacy of the internet of things to be reduced in consideration of the networks and this approach made a efficiency high.the main issues the network boundary and load balancing are not into consideration.the internet of things nodes are controlling in power process and energy.

Kharrufa et al. [17] presented a routing algorithm for lossy network and low power which can be used in RPL enhancement for the network to be dynamically changing with various networks. The packet delivery ratio, reasonable energy and end to end delay are enhanced by RPL routing protocol.

Kim el al. [18] proposed the RPL issue which are congestion and load balancing. The traffic issue made the packet loss and to solve this issue ,the RPL Queue Utilization is achieved with taking the neighbour nodes into consideration and that led to Queue loss to be prevented from loss and made the increased of the PDR.

\section{ARCHITECTURE OF THE INTERNET OF THINGS}

As of now The IOT Architecture has no specific architecture, each researcher explain different Architecture and the main concept to be in the consideration is the three layer architecture concept which are the object layer ,perception layer ,network layer and application layer are the main layer Architecture for the IOT.

Three- layer Architectures

The figure -2 show the main basic layer architecture which many research explained about it in the early years in the area of the internet of things .there are three layers which are as ,Application layer, Network layer and Perception layer. The network layer is one of the most important layer that can be connected to various networks devices, smart devices and also servers. The Application layer is mainly used for applications 
delivering and many services to the clients, also used for different application in order to be deployed by the internet of things.the third layer is the perception layer which has sensors and can be used as physical layer that can be able for gathering and sensing data information of the environment.it used for sensing the parameters physically and the identifiers of other environment objects.

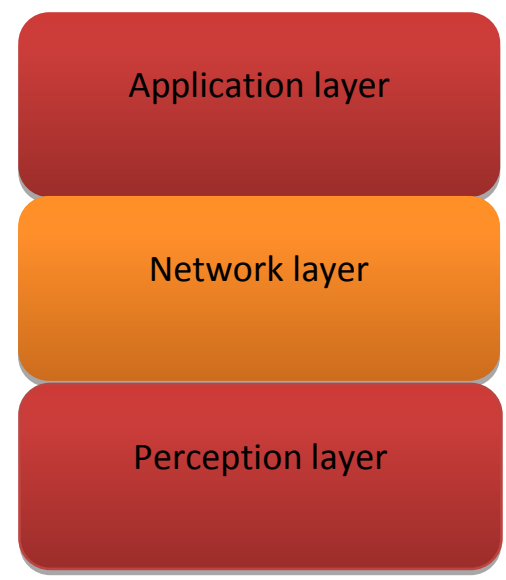

Fig 2: Architecture layers

This layers are the main layers that can be consider to be used in the internet of things architecture. There are many research that are searching for proper framework of the internet things because this three layer are not enough as the efforts of the research are the internet of things aspect .The perception and layer and the Application layer are the most consider layer for the IOT.it helps to manage and make a suitable services to other layers[19].

\section{FEATURES OF THE INTERNET OF THINGS}

Internet of things (IOT) Features is one of the main part of the IOT field which enables the use of the internet of things devices. Here are some features that can be described as following [20]:

1) Things: the things are the object that are connected to each other in order to perform a task the objects can be as home appliances, mobile, actuator, sensor etc that are connect to an Internet of things object this objects devices are the main crucial for the Internet of things in order to make the use of the IOT technology.

2) Data: the Data are also one of the important concerns of the internet in order to perform a proper task in the field of the IOT. The devices are connected to each other and needs to share the data between them that led to the internet of things network to handle such a huge amount of the data. Also the management of the data tools are useful for the internet of things network.

3) Connectivity: Internet of things connectivity is one of the features that lead to connect networks devices to each other in order to produce and access the data.

4) Intelligence: The analysing of the data using though the sensing of the ability devices in the networks internet of things.the algorithms are one of the most used for the data analyzing.

5) Communication: the communication in IOT field is also a big feature that is used for various devices which will be connected each other in order to make a proper communication with each devices. Once the communication is done to each device there is a way of access of the data which can be analysis.

\section{CHALLENGES OF THE INTERNET OF THINGS}

Internet of things are one of the most important challenges for the researchers. Especially when it comes to the security, designing, in the field of industry, architecture of the internet of things, developing the business of the internet of things model, etc ,that brings the internet of thins is more useful technology which connect many objects to each other in order to transfer the data from source to destination securely

Many researcher are try to develop a protocol or techniques that can be suitable for the internet of things which can be led to make a proper framework of the internet of things .so this is another challenges of the researcher in order to achieve a proper framework technology for the IOT[21].

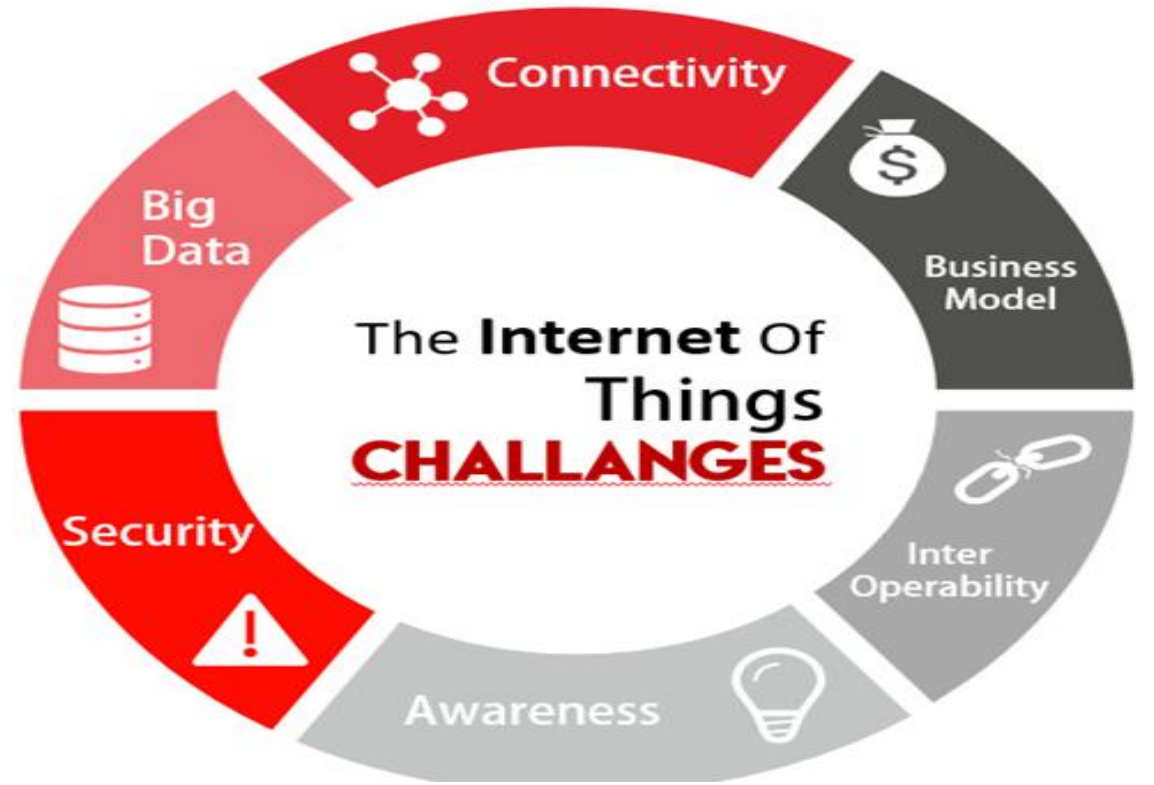

Fig 3: Internet of things challenges 
The figure 3 illustrate the challenges of the internet of things which are include the connectivity issue ,interoperability ,security ,Awareness , Big data, business model etc. This challenges are the most important challenges in order to make use of the internet of things devices

\section{STATUS OF THE INTERNET OF THINGS}

The internet of things are rapidly increased day by day and .As the covid-19 pandemic happened there more growing of the IOT devices to be connected to each other's the 2020 year made the growth the internet more useful for the first time ,there are many devices of the internet of things connection for example smart home, health care, industries ,smart appliance etc, According to the statics there will be many devices of the internet of things connected in 2020.Also there are many research expected that many devices going to be connected by 2025 which approximately going to be more than 30 billion internet of things devices connection and that will make each person to have at least 4 internet of things devices on average.

\section{Total number of device connections (incl. Non-loT)}

\section{$20.0 \mathrm{Bn}$ in 2019 - expected to grow $13 \%$ to $41.2 \mathrm{Bn}$ in 2025}

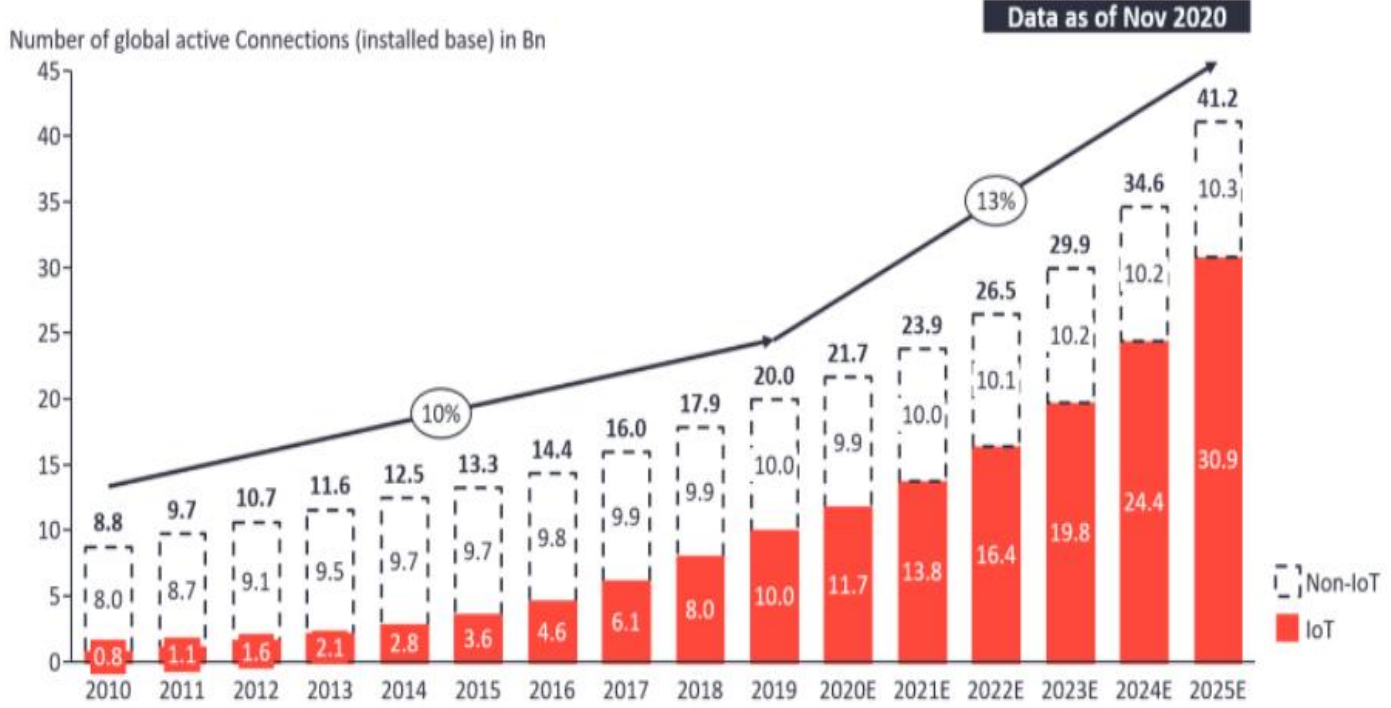

Fig 4: status of internet of things

According to the analysis done in last two years, internet of things analytics have been growing up its prediction for the many connected devices in 2025 and that going to average from 21.5 billion to 30 billion .Many elements are leading the raised of the IOT devices which notably:

China: The internet of things rapidly increased in china as many devices has been connected to each other comparing to few years ago .Especially till 2015 Chinese companies still just the connection devices only $27 \%$ for all cellular internet of things .in the recent years the number of IOT devices is increased in China by more than $75 \%$ in 2020 .the China Unicom and Telecom are the most used for the IOT devices .The market in china are supporting by the cellular IOT connections.

Home devices. The extraordinary use of the internet of things devices which are wearables in last 2 to 3 years and which is going to be continues furthermore and that led to rapidly increase of smart devices to be connected in homes which made the possibility of the internet of things to be expected more in the coming years.

The low-power wide-area(LPWA) or Low-power wide-area network(LAWAN): this technology is one of the most type of WSN communication that are used for allowing good communication with minimum rate cost among the connected objects which are the actuators, sensors smart cities etc. The Lower-wide area network were just about 10 million connections devices in the year of 2015.in the upcoming years ,this technology enables many connections of the internet of things devices which is a remote battery devices for example containers in logistic ,home appliance, smarts cities etc. The growth in 2020 has been increased to more than 423 million connected devices and its more expected to be rapidly increase to 2.5 million connection devices of the IOT by 2025 .taking into consideration of $5 \mathrm{G}$ technology there might be more IOT connection in the future[22].

\section{CONCLUSION}

As the internet of things is growing day by day in daily life, this technology going to make the life easier by connected several devices and Applications. However the internet of things will make everything's around us to be connected and to be smart. The IOT can be connected many objects to each other in order to send the data from source to destination. in the recent years the researcher have been quickly increased in the term of smart cities „,health care, industrial automation, environmental monitoring for utilizing the internet of things which basically for efficient data transmission and gathering. Hence, it's more important to perform a multipath routing protocol in order to overcome the issue of the energy constraint and connectivity of the networks. This paper presents the proficient empirical optimization techniques and routing protocols that can be used in the interne of things fields.

In future work will be focusing on the selection of best 
routing protocol that can be used in the internet of things field and optimization problem to increase the parameters using advanced optimization techniques. In Another way for future research is to extend the existing system model in order to achieve best routing protocol for internet of things.

\section{REFERENCES}

[1] S. Park, N. Crespi, H. Park and S. H. Kim, "IoT routing architecture with autonomous systems of things," In Proc. 2014 IEEE World Forum on Internet of Things (WF-IoT), pp. 442-445, March 2014.

[2] R. Fantacci, T. Pecorella, R. Viti and C. Carlini, "A network architecture solution for efficient IoT WSN backhauling: challenges and opportunities," IEEE Wireless Communications, vol. 21, no. 4, pp. 113-119, 2014.

[3] P. Rajpoot, S. H. Singh, R. Verma, K. Dubey, S. K. Pandey and S. Verma, "Multi-factor-Based EnergyEfficient Clustering and Routing Algorithm for WSN," In Soft Computing: Theories and Applications, Springer, Singapore, pp. 571-581, 2020.

[4] S. Mohapatra and P. Kanungo, "Performance analysis of AODV, DSR, OLSR and DSDV routing protocols using NS2 Simulator," Procedia Engineering, vol. 30, pp. 6976, 2012.

[5] Z. Fei, B. Li, S. Yang, C. Xing, H. Chen and L. Hanzo, "A survey of multi-objective optimization in wireless sensor networks: Metrics, algorithms, and open problems," IEEE Communications Surveys and Tutorials, vol. 19, no. 1, pp. 550-586, 2016.

[6] M. Radi, B. Dezfouli, K. A. Bakar and M. Lee, "Multipath routing in wireless sensor networks: survey and research challenges," Sensors, vol. 12, no. 1, pp. 650-685, 2012.

[7] Pan, M. S., \& Yang, S. W. (2017). A lightweight and distributed geographic multicast routing protocolfor IoT applications. Computer Networks, 112, 95-107.

[8] Rani, S., Talwar, R., Malhotra, J., Ahmed, S. H., Sarkar, M., \& Song, H. (2015). A novel scheme for anenergy efficient internet of things based on wireless sensor networks. Sensors, 15(11), 28603-28626.

[9] Tseng, C. H. (2016). Multipath load balancing routing for internet of things. Journal of Sensors. https://doi.org/10.1155/2016/42507 46.

[10] Qiu, T., Liu, X., Feng, L., Zhou, Y., \& Zheng, K. (2016). An efficient tree-based self-organizing protocol for internet of things. IEEE Access, 4, 3535-3546.

[11] Shen, J., Wang, A., Wang, C., Hung, P. C., \& Lai, C. F. (2017). An efficient centroid-based routing protocol for energy management in WSN-assisted IoT. IEEE Access, $5,18469-18479$.

[12] Baker, T., Asim, M., Tawfik, H., Aldawsari, B., \& Buyya, R. (2017). An energy-aware service composition sition algorithm for multiple cloud-based IoT applications. Journal of Network and Computer Applications, 89, 96-108.

[13] Qiu, T., Lv, Y., Xia, F., Chen, N., Wan, J., \& Tolba, A. (2016). ERGID: An efficient routing protocol for emergency response internet of things. Journal of
Network and Computer Applications, 72, 104-112.

[14] Xie, J., Lyu, L., Deng, Y., \& Yang, L. T. (2015). Improving routing performance via dynamic programming in large-scale data centers. IEEE Internet of Things Journal, 2(4), 321-328.

[15] Hasan, M. Z., \& Al-Turjman, F. (2017). Optimizing multipath routing with guaranteed fault tolerance in internet of things. IEEE Sensors Journal, 17(19), 6463 6473 .

[16] Shu, T., \& Cui, S. (2017). Renovating location-based routing for integrated communication privacy and efficiency in IoT. In IEEE international conference on communications (ICC) (pp. 1-6). IEEE.

[17] Kharrufa, H., Al-Kashoash, H., Al-Nidawi, Y., Mosquera, M. Q., \& Kemp, A. H. (2017). Dynamic RPL for multi-hop routing in IoT applications. In 13th Annual conference on wireless on-demand network systems and services (WONS) (pp. 100-103). IEEE.

[18] Kim, H. S., Kim, H., Paek, J., \& Bahk, S. (2017). Load balancing under heavy traffic in RPL routing protocol for low power and lossy networks. IEEE Transactions on Mobile Computing, 16(4), 964-979.

[19] Pallavi Sethi and Smruti R. Sarangi(2017) Internet of Things: Architectures, Protocols, and Applications. Journal of Electrical and Computer Engineering Volume 2017, Article ID 9324035

[20] Syed Yasmeen Shahdad, Mudassir Khan, Habeeba Sultana, Mohammad Ashfaq Hussain and Syeda Meraj Bilfaqih(2019) Routing Protocols for Constraint Devices in an Internet of Things Network. International Conference on Communication and Signal Processing.

[21] Peter Ryan, Richard Watson Richard Watson (2017), Research Challenges for the Internet of Things: What Role Can OR Play? https://www.researchgate.net/publication/315058460

[22] State of the IoT 2020: 12 billion IoT connections, surpassing non-IoT for the first time.https://iot analytics.com/state-of-the-iot-2020-12-billion-iotconnections-surpassing-non-iot-for-the-first-time/

\section{AUTHOR'S PROFILE}

Mr.Salem Abdulla Awadh Saeed Bahmaid received his BCA from St. Philomena's College, Mysore, and M.IT from Dr G.R. Damodaran College of Science, Coimbatore. Presently he is pursuing his $\mathrm{PhD}$ in the field of Advance networking at Rathinam College of Arts and Science, Bharathiar University, Coimbatore, India. His research interests focus on Computer Networks and IoT.

Dr.V.Vasanthi pursed M.Sc (CS), M.Phil. And PhD. Computer Science from Karpagam University, Coimbatore in 2014. She is currently working as Professor in the Department of Computer Science from the Department of Information Technology, Sri Krishna Adithya College of Arts And Science, Bharathiar University, Coimbatore. She has published more research papers in reputed international journals including Thomson Reuters (SCI \& impact factor) and conferences including IEEE \& Springer and it's also available online. Her main research work focuses on Ad-hoc and Sensor Networking. 